\title{
Human death effector domain-associated factor interacts with the viral apoptosis agonist Apoptin and exerts tumor-preferential cell killing
}

\author{
AAAM Danen-van Oorschot ${ }^{1,2}$, P Voskamp ${ }^{1}$, MCMJ Seelen ${ }^{1}$, \\ MHAM van Miltenburg ${ }^{1}$, MW Bolk ${ }^{1}$, SW Tait ${ }^{3}$, JGR Boesen-de \\ Cock $^{3}$, JL Rohn ${ }^{1}$, J Borst ${ }^{3}$ and MHM Noteborn*,1,2 \\ ${ }^{1}$ Leadd BV, Leiden, The Netherlands; \\ 2 BFSC-Leiden Institute of Chemistry, Leiden University, Leiden, \\ The Netherlands; \\ ${ }^{3}$ Division of Cellular Biochemistry, The Netherlands Cancer Institute, \\ Amsterdam, The Netherlands \\ * Corresponding author: MHM Noteborn, Leiden University, PO Box 9502, \\ Leiden 2300 RA, The Netherlands. Tel: + 3171 5274414; \\ fax : + 3171 5274357; E-mail: m.noteborn@chem.leidenuniv.nl
}

Received 23.5.03; revised 02.12.03; accepted 09.12.03; published online 6.2.04 Edited by Dr. JM Hardwick

\begin{abstract}
Apoptin, a protein from chicken anemia virus without an apparent cellular homologue, can induce apoptosis in mammalian cells. Its cytotoxicity is limited to transformed or tumor cells, making Apoptin a highly interesting candidate for cancer therapy. To elucidate Apoptin's mechanism of action, we have searched for binding partners in the human proteome. Here, we report that Apoptin interacts with DEDAF, a protein previously found to associate with death effector domain (DED)-containing pro-apoptotic proteins, and to be involved in regulation of transcription. Like Apoptin, after transient overexpression, DEDAF induced apoptosis in various human tumor cell lines, but not in primary fibroblasts or mesenchymal cells. DEDAF-induced cell death was inhibited by the caspase inhibitor p35. Together with the reported association of DEDAF with a DED-containing DNA-binding protein in the nucleus and the transcription regulatory activity, our findings may provide a clue for the mechanism of Apoptin's actions in mammalian cells.

Cell Death and Differentiation (2004) 11, 564-573.

doi:10.1038/sj.cdd.4401391

Published online 6 February 2004
\end{abstract}

Keywords: apoptin; apoptosis; DEDAF; RYBP; transcriptional repression; YY1

Abbreviations: CAV, chicken anemia virus; DAPI, 2,4-diamidino-2-phenylindole; DED, death effector domain; DEDD, DEDcontaining DNA-binding protein; DEDAF, death effector domainassociated factor; FADD, Fas-associated death domain; Gal4 BD, Gal4 DNA-binding domain; NLS, nuclear localization signal; PcG, Polycomb group; RYBP, Ring1- and YY1-binding protein; UBF, upstream-binding factor; YAF2, YY1-associated factor 2; YEAF1, YY1- and E4TF1/hGABP-associated factor

\section{Introduction}

Cancer therapy using chemotherapeutics or ionizing radiation is based in part on the ability of these regimens to kill tumor cells by apoptosis. However, during tumorigenesis, cells accumulate mutations that thwart apoptosis, such as loss of functional p53 or overexpression of Bcl-2. ${ }^{1}$ Unfortunately, these genetic alterations are likely to increase resistance to chemotherapy or radiation, especially in hematopoietic cells. $^{2,3}$ Therefore, apoptotic agents that can circumvent these mutations would serve as attractive candidates for cancer therapy. Apoptin, a gene product of chicken anemia virus (CAV), is such a molecule: in contrast to many conventional approaches, Apoptin-induced apoptosis is independent of functional p53, and is not inhibited, but is even accelerated, by overexpression of $\mathrm{Bcl}-2 .{ }^{4}$

Apoptin, or VP3, is a small protein of 121 amino acids, rich in prolines, serines, and threonines, with a bipartite-type nuclear localization signal (NLS) and a hydrophobic sequence resembling a nuclear export signal. No recognizable homologues have been found in any species. Expression of Apoptin is sufficient to induce apoptosis in transformed chicken thymocytes in culture,$^{5}$ as well as in transformed or tumor cells of human origin. ${ }^{6}$ Apoptin induces typical apoptotic nuclear morphology, DNA laddering, and release of cytochrome c. Furthermore, Apoptin-induced cell death is inhibited by the caspase inhibitor p35. ${ }^{7}$ Together, these observations indicate that Apoptin-induced cell death occurs by apoptosis. Remarkably, overexpression studies in over 70 tumor cell lines and over 10 normal cell types indicate that Apoptin induces apoptosis specifically in transformed or tumor cells. ${ }^{8-10}$ Normal cells become susceptible upon spontaneous or SV40 virus-induced transformation. Moreover, in transient transfections, co-expression of Apoptin with SV40 large T antigen induced apoptosis in normal diploid fibroblasts. ${ }^{9,11}$ These data indicate that the ability of Apoptin to induce apoptosis coincides with the transformed state of a cell or a transformation-specific condition, making it an even more exceptional candidate for cancer therapy.

The tumor-specific activity of Apoptin seems to be related to its differential localization. In tumor cells, Apoptin is present predominantly in the nucleus, whereas in healthy, untransformed cells, it localizes mainly in the cytoplasm. ${ }^{8}$ Mutational analyses indicate that nuclear localization is required, but not sufficient, for the pro-apoptotic activity of Apoptin in tumor cells. $^{12,13}$ These data suggest that the tumor-specific activity of Apoptin is not caused by nuclear localization per se. Rather, differential phosphorylation was found to be involved; ${ }^{14}$ furthermore, binding to factors that are expressed at higher levels in transformed cells than in normal diploid cells may also play a role.

At present, the mechanism of action of Apoptin is unclear. In particular, the lack of cellular homologues has prevented 
genetic approaches to elucidate its function. Therefore, we searched for binding partners of Apoptin in the hope of providing important information about its molecular mechanism of tumor-specific apoptosis induction. Here, we identify Death Effector Domain-associated factor (DEDAF) as a new binding partner of Apoptin that, like Apoptin, induces apoptosis specifically in tumor cells.

\section{Results}

\section{Apoptin interacts with human DEDAF}

To learn more about the mechanism by which Apoptin induces apoptosis in human cells, we searched for human Apoptinbinding proteins using yeast two-hybrid screens. Full-length Apoptin fused to the GAL4 DNA-binding domain was used as bait. Expression of this fusion protein in reporter yeast cells revealed that Apoptin does not activate gene transcription by itself in a yeast background (data not shown). The Apoptin construct was co-expressed with a cDNA library derived from Epstein-Barr virus-transformed human B lymphocytes. ${ }^{15}$ Approximately $1 \times 10^{6}$ colonies were screened for growth on medium lacking $\mathrm{His}$, and positive clones were tested for $\beta \mathrm{Gal}$ activity in two additional rounds of selection. Among the resulting positive clones, two contained cDNAs encoding the same protein, which we initially termed Apoptin-associating protein 1 (AAP1). Recently, this protein was identified by another group as DEDAF, ${ }^{16}$ and it has also been named YEAF1 (YY1- and E4TF1/hGABP-associated factor). ${ }^{17}$ One of our clones contained the complete open reading frame (GenBank \# AY228125). The other clone encoded DEDAF with an N-terminal deletion of 30 amino acids, and was designated DEDAF- $\triangle \mathrm{N} 30$.

DEDAF is the human homologue of murine RYBP (Ring1and YY1-binding protein), which was isolated in a yeast twohybrid screen for binding partners of the Polycomb group of transcription regulators. ${ }^{18}$ Inspection of the human genome databases revealed that DEDAF and RYBP have one relative, namely YAF2 (YY1-associated factor 2). ${ }^{19}$ Like RYBP, the DEDAF cDNA contains two ATG codons at the $5^{\prime}$ end, of which the second lies in an optimal Kozak consensus sequence $^{20}$ and aligns with the start codon of YAF2. Both DEDAF cDNAs isolated in the yeast-two hybrid screen contain a stop codon that aligns with the stop codons of RYBP and YAF2, resulting in a predicted protein of 226 amino acids. Searches of EMBL/GenBank databases provided a number of human expressed-sequence tag (EST) clones that extended the $3^{\prime}$ end to approximately $3.5 \mathrm{~kb}$, but did not extend the $5^{\prime}$ end of the DEDAF cDNAs significantly more. There are no apparent coding regions in the large $3^{\prime}$ end.

To determine whether DEDAF associates with Apoptin in a mammalian cell line, we performed co-immunoprecipitation experiments in Cos- 1 cells. To this end, cells were transfected with plasmids encoding myc-tagged DEDAF or DEDAF- $\triangle N 30$ and cotransfected with Apoptin or an empty vector. As an additional negative control, Apoptin was co-expressed with myc-tagged LacZ. Complexes were precipitated from detergent lysates with anti-myc antibody to detect DEDAF or LacZ, or antibody R $\alpha$ VP3-C directed against the C-terminus of Apoptin. The complexes were analyzed by Western blotting with the same anti-myc mAb and a second antibody specific for Apoptin. Apoptin co-immunoprecipitated with full-length DEDAF (Figure 1), indicating that it can bind DEDAF in mammalian cell lysates. The deletion of amino acids 1-30 in DEDAF- $\Delta \mathrm{N} 30$ did not prevent binding to Apoptin, indicating that the putative zinc-finger at amino acids $25-42$ is not involved in this interaction (Figure 1). DEDAF and DEDAF$\triangle \mathrm{N} 30$ did not co-immunoprecipitate with anti-Apoptin antibody, suggesting that either the epitope recognized by antibody R $\alpha$ VP3-C (76-90 aa) is involved in DEDAF binding, or binding of this antibody results in steric hindrance. Apoptin did not co-immunoprecipitate with myc-tagged LacZ, confirming the specificity of the Apoptin/DEDAF interaction. Thus, DEDAF not only binds to Apoptin in the yeast two-hybrid system, but also in a mammalian background.

\section{DEDAF is widely expressed in adult and fetal tissues at the RNA level}

It has previously been reported that DEDAF is ubiquitously expressed in human tissues with a particular abundance in placenta and hematopoietic organs. ${ }^{16}$ Consistent with these data, we detected a product of approximately $4.5 \mathrm{~kb}$ at low abundance in multiple tissues and relatively high levels in placenta by Northern blotting of polyA ${ }^{+}$RNA (Figure 2). Expression in thymus and spleen, however, was similar to that in most other tissues. Analysis of mRNA from tumor cell lines revealed broad expression of the $4.5 \mathrm{~kb}$ transcript and also did

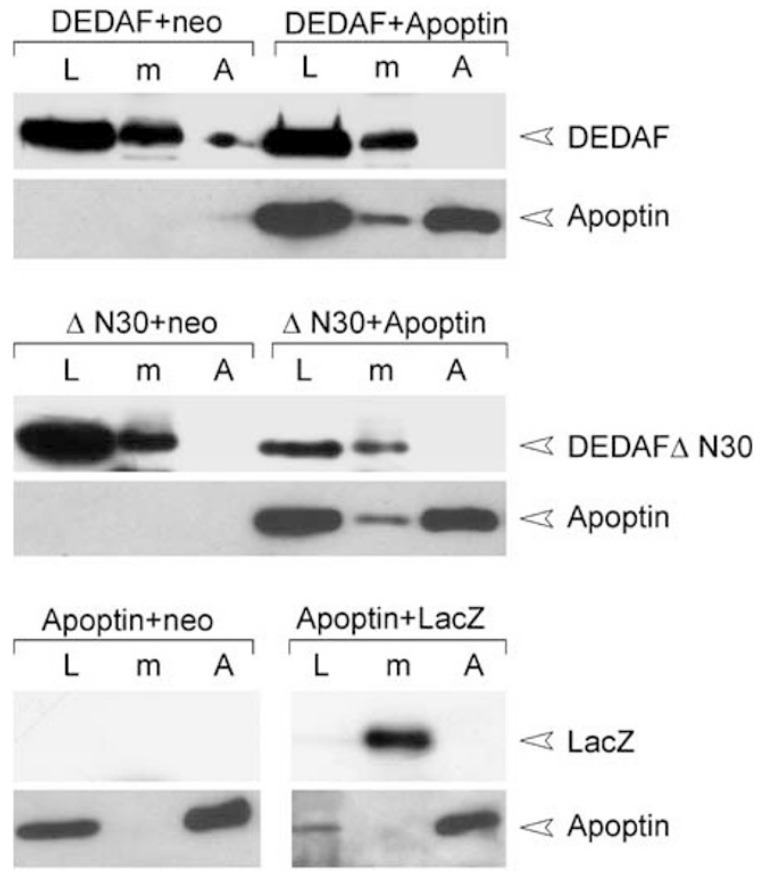

Figure 1 DEDAF and DEDAF- $\triangle N 30$ interact with Apoptin in mammalian cells. Cos-1 cells were transfected with plasmids encoding myc-tagged DEDAF, DEDAF- $\Delta \mathrm{N} 30(\Delta \mathrm{N} 30)$, or LacZ, untagged Apoptin, or empty plasmid (neo) in the indicated combinations. Total lysates $(L)$ or protein complexes immunoprecipitated with mAb 9E10 directed against the myc-tag $(\mathrm{m})$ or antibody R $\alpha \mathrm{VP} 3-\mathrm{C}$ directed against Apoptin (A) were separated by SDS-PAGE, and analyzed by Western blot. Myc-tagged proteins were detected with anti-myc mAb 9E10, and Apoptin was detected with antibody CVI-CAV-111.3 
a

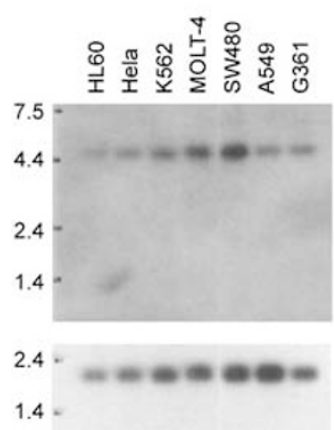

b

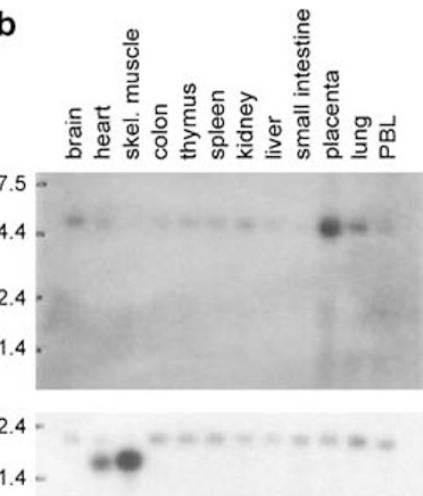

C

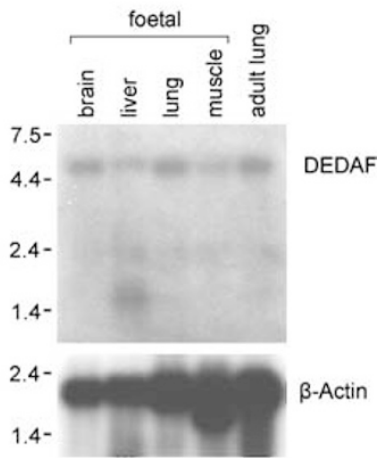

Figure 2 DEDAF is encoded by a ubiquitous $4.5 \mathrm{~kb}$ transcript with high expression in placenta. All the three Northern blots were probed first with DEDAF cDNA (top, DEDAF), then stripped and re-probed with $\beta$-actin CDNA (bottom) as a control for equal loading. (a) Poly $\mathrm{A}^{+}$RNA from human tumor cell lines, namely HL60 (promyelocytic leukemia), HeLa (cervix carcinoma), K562 (chronic myelogenous leukemia), MOLT4 (lymphoblastic leukemia), SW480 (colorectal adenocarcinoma), A549 (lung carcinoma), G361 (melanoma). (b) Poly A ${ }^{+}$RNA from various normal, adult tissues. (c) Total RNA from four different fetal tissues from 22-37-week-old embryos, and adult lung. Markers indicate sizes in $\mathrm{kb}$

not show increased expression in lines of hematopoietic origin, nor the presence of alternative transcripts. The abundance of DEDAF transcript in placenta suggested that the DEDAF gene might also be highly transcribed in fetal tissues. However, Northern blotting of polyA ${ }^{+}$RNA from the fetal brain, liver, lung, muscle, and adult lung revealed that all the four fetal tissues contained levels of DEDAF mRNA comparable to that of adult lung, but not as high as that in placenta. Two smaller transcripts of about 1.5 and $2.4 \mathrm{~kb}$ were seen in fetal liver. These results indicate that DEDAF is expressed as a $4.5 \mathrm{~kb}$ transcript at similar low levels in a broad range of adult and fetal tissues, and has a strikingly high expression in placenta.

\section{DEDAF binds to YY1 and acts as a repressor in a reporter assay}

DEDAF can be considered the human homologue of murine RYBP, a protein that has been implicated thus far not in apoptosis, but in regulation of transcription. In addition, DEDAF is highly homologous to YAF2, which shares with RYBP the capacity to interact with and regulate the activity of the YY1 transcription factor. ${ }^{18,19}$ Upon transfection into Cos-1 cells, myc-tagged DEDAF was found to interact with HAtagged human YY1. The interaction was not affected by deletion of the $30 \mathrm{~N}$-terminal amino acids of DEDAF (Figure 3a). In cells in which only DEDAF was expressed, the YY1 antibody immunoprecipitated some DEDAF. This can be explained by binding of DEDAF to endogenous YY1.

To test whether DEDAF can regulate transcription, we established a reporter assay. The plasmid $5 \times$ Gal4-TK-Luc, containing the luciferase gene, a thymidine kinase (TK) TATA promoter, and a Gal4 upstream activating sequence was used as a reporter. This reporter gives a high basal level of luciferase activity, which can be either enhanced or repressed by factors tethered to the promoter via the Gal4 DNA-binding domain. Cotransfection of the reporter plasmid with Gal4YY1, known to repress transcription in a similar system, ${ }^{21}$ resulted in repression of luciferase activity (Figure $3 b$ ). Likewise, DEDAF fused to the Gal4 DNA-binding domain repressed luciferase acitivity, even when as little as $2 \mathrm{ng}$ of plasmid was added (Figure $3 b$ ). These data suggest that one of the functions of DEDAF in vivo is to repress transcription and thus regulate gene expression. Whether this can be accomplished by influencing $\mathrm{YY} 1$ activity has yet to be determined.

\section{DEDAF is predominantly nuclear and partially co-localizes with Apoptin in intact and apoptotic tumor cells, but not in normal cells}

Apoptin has a characteristic intracellular localization. Upon overexpression into healthy, untransformed human cells, it is mainly cytoplasmic. In transformed cells, however, it assumes a predominantly nuclear localization, which is likely required for its pro-apoptotic activity. ${ }^{8,12,13}$ We examined whether the subcellular distribution of overexpressed DEDAF was consistent with a functional interaction with Apoptin in intact cells. When expressed alone in Saos-2 osteosarcoma cells, DEDAF was to some extent cytoplasmic, but the great majority of staining was found in the nucleus, distributed evenly in a fine granular pattern, as seen by fluorescent and confocal microscopy (Figure 4j-k). Occasionally, it was detected in perinuclear structures and more strongly staining, round subnuclear bodies. Cotransfection with Apoptin did not alter the localization of DEDAF (Figure 4), whereas DEDAF did influence the localization of Apoptin; namely, Apoptin was more prominently present in the cytoplasm when DEDAF was co-expressed. In about $25 \%$ of cotransfected cells, DEDAF co-localized almost completely with Apoptin in the nucleus, often with both proteins being visible in the same subnuclear bodies (Figure 4a-c). DEDAF showed partial co-localization with Apoptin in the majority of cells (about 75\%), as shown in Figure $4 \mathrm{~d}-\mathrm{f}$. In a number of co-expressing cells, DEDAF was present only in the nucleus, while Apoptin was found both in the nucleus and in the cytoplasm. In apoptotic cells, DEDAF and Apoptin were both detected predominantly in the nucleus, but their distribution within the nucleus only partially overlapped (Figure 4g-i). DEDAF- $\triangle \mathrm{N} 30$ showed essentially the same localization as wild-type DEDAF, both in the presence 
a

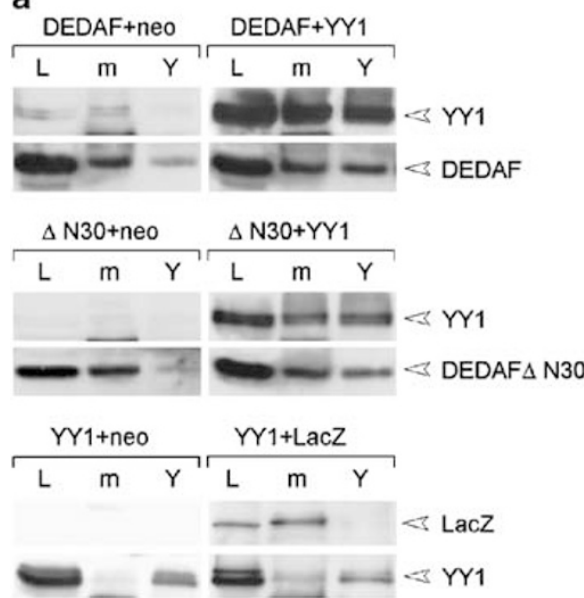

b

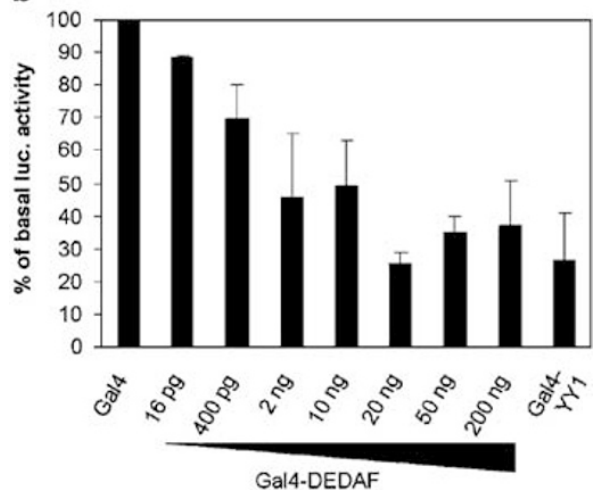

Figure 3 DEDAF interacts with $Y Y 1$ and similarly represses gene transcription (a) Cos-1 cells were transfected with plasmids encoding myc-tagged DEDAF, DEDAF- $\triangle N 30$, or LacZ, HA-tagged $Y Y 1$, or an empty control (neo), in the indicated combinations. Total lysates $(\mathrm{L})$ or protein complexes immunoprecipitated with anti-myc $m A b(m)$ or polyclonal serum directed against $Y Y 1(Y)$ were separated by SDS-PAGE and analyzed by Western blot. Myc-tagged proteins were detected with anti-myc $\mathrm{mAb}$, and $\mathrm{YY} 1$ with an antibody directed against the HA-tag. (b) U2OS cells were transfected with a decreasing amount of plasmid encoding DEDAF fused to the Gal4 BD, $200 \mathrm{ng}$ of plasmid encoding YY1 fused to Gal4 BD, or Gal4 BD alone as a control for basal activity, and the TK-TATA luciferase plasmid as a reporter. A LacZ plasmid was co-transfected as a control for transfection efficiency. At day 2 post-transfection, lysates were made and analyzed for $\beta$-Gal and luciferase activity. The control, Gal4 BD, was set to $100 \%$. Values shown are normalized for $\beta$-Gal, and represent three independent experiments, each performed in duplicate

and absence of Apoptin, indicating that the $30 \mathrm{~N}$-terminal amino acids are not essential for its localization (data not shown).

As reported earlier, in normal cells, Apoptin was localized preferentially in the cytoplasm. ${ }^{8}$ This is shown for fibroblasts and mesenchymal stem cells in Figure 5 . In these cells, DEDAF exhibited a predominantly nuclear localization, as in tumor cells. Co-expression with Apoptin did not affect this localization (Figure 5). DEDAF distribution was homogenous throughout the nucleus, although round areas of negative staining were regularly observed. In conclusion, DEDAF and Apoptin partially co-localize in the nucleus of tumor cells, Apoptin does not affect the localization of DEDAF, and these proteins are differentially distributed in normal cells.
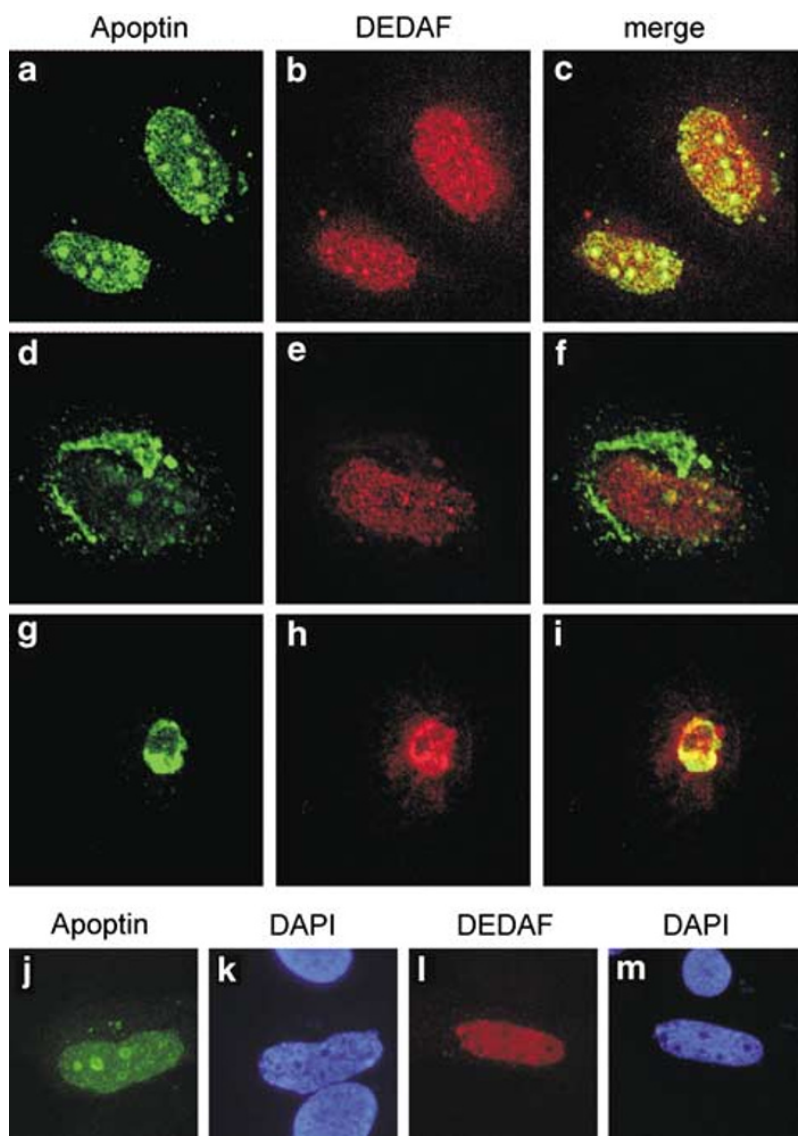

DAPI
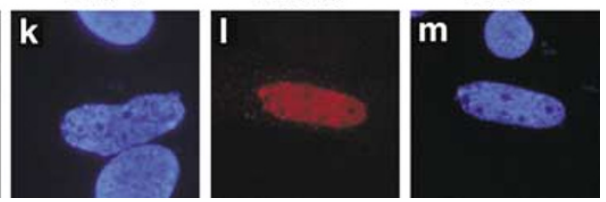

Figure 4 DEDAF is nuclear and partially co-localizes with Apoptin in tumor cells. Saos-2 cells were co-transfected with plasmids encoding DEDAF and Apoptin, and stained by indirect immunofluorescence for Apoptin (green; $\mathbf{a}, \mathbf{d}, \mathbf{g}$ ), and DEDAF (red; $\mathbf{b}, \mathbf{e}, \mathbf{h}$ ); merged images are also shown (c, $\mathbf{f}, \mathbf{i})$. Pictures were made by confocal imaging (3D reconstruction). (a-c) Images representative of about $25 \%$ of cells examined. DEDAF co-localizes almost completely with Apoptin. (d-f) Images representative of about $75 \%$ of transfected cells examined. DEDAF partially co-localizes with Apoptin. (g-i) Partial co-localization was also found in apoptotic cells. (j-m) illustrate the localization of Apoptin (green; $j$ ) or DEDAF (red; I) alone in Saos-2 cells. Nuclei were counterstained with DAPI (blue; $\mathbf{k}, \mathbf{m}$ ), images were made by fluorescence microscopy

\section{Apoptin is enriched in nucleoli, but DEDAF is not}

When Apoptin was expressed alone, or together with DEDAF, in some cells Apoptin was enriched in nuclear bodies that resemble nucleoli in size and number, and which excluded DAPI staining. To determine if these bodies were indeed nucleoli, we examined Saos-2 cells for co-localization of ectopically expressed Apoptin and/or DEDAF with an upstream-binding factor (UBF), a basal factor for RNA polymerase I transcription that is exclusively located in the nucleoli. ${ }^{22}$ This experiment showed that the brightly stained Apoptin bodies in the nucleus clearly co-localized with UBF, which suggests that Apoptin is present in significant amounts in nucleoli (Figure 6a-c). However, DEDAF, either alone or in the presence of Apoptin, did not co-localize with UBF, although in some occasions it was observed in close proximity (Figure 6d-f). Nuclear bodies were not always visible in cells transfected with DEDAF; however, in those cells in which bodies were visible, no co-localization with UBF was seen. 


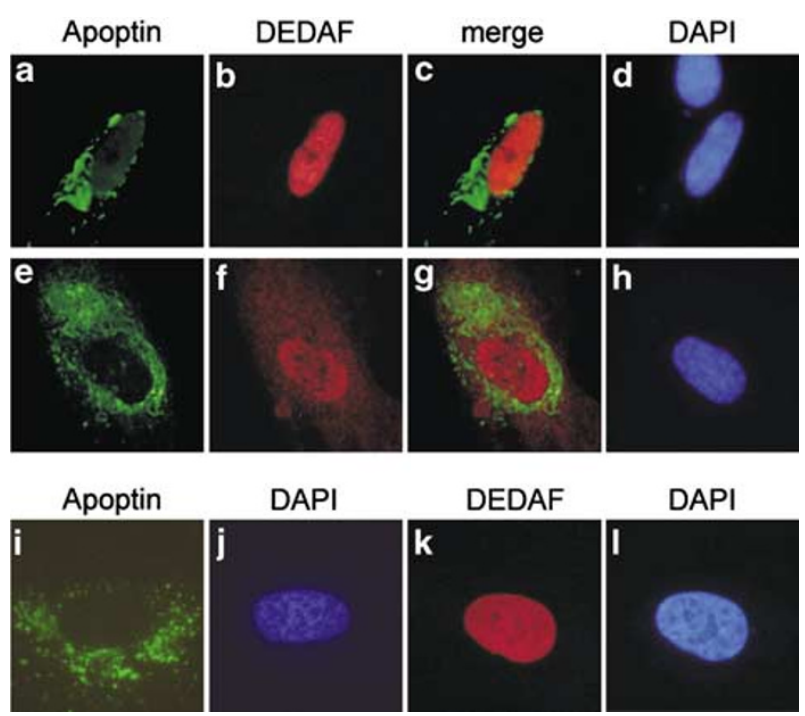

Figure 5 DEDAF shows a different intracellular distribution than Apoptin in normal cells. Diploid foreskin fibroblasts (a-d) and mesenchymal stem cells $(\mathbf{e}-\mathbf{h})$ were microinjected with plasmids encoding Apoptin and DEDAF. Expression of these proteins was detected by indirect immunofluorescence for Apoptin (green; $\mathbf{a}, \mathbf{e})$ and DEDAF (red; $\mathbf{b}, \mathbf{f})$. Merged images are shown in (c) and (g). (i-l) show the expression pattern of either Apoptin (green; $i)$ or DEDAF (red; $\mathbf{k}$ ) alone in diploid foreskin fibroblasts. Nuclei were counterstained with DAPI (blue; $\mathbf{d}, \mathbf{h}, \mathbf{j}, \mathbf{l}$ ), images were made by fluorescence microscopy
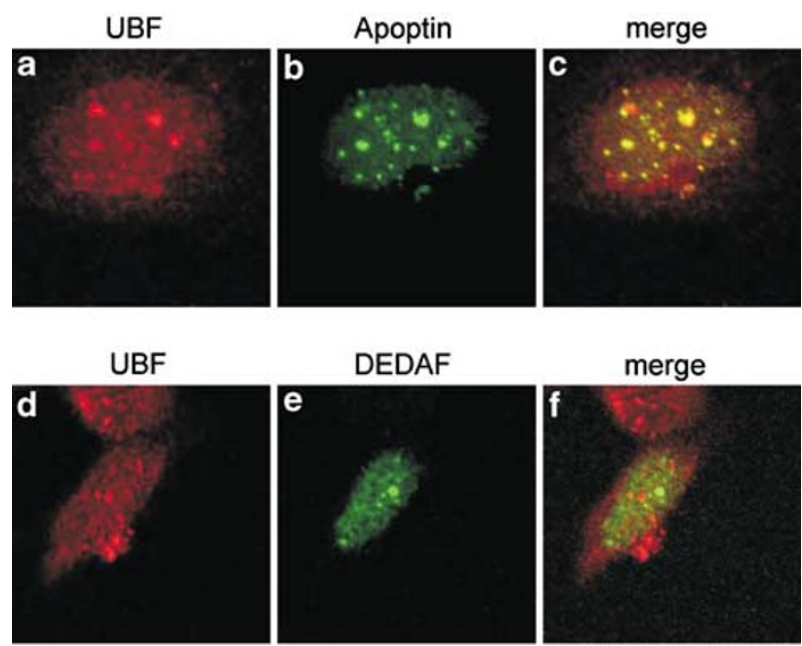

Figure 6 Apoptin co-localizes with UBF in nucleoli, but DEDAF does not. Saos2 cells were transfected with plasmids encoding Apoptin or DEDAF, and stained by indirect immunofluorescence for UBF (red) and Apoptin (green) in (a-c), with (c) showing the merged image, or for UBF (red) and DEDAF (green) in (d-f), with (f) showing the merged image. Pictures were made by confocal imaging (3D reconstruction)

Thus, Apoptin, but not DEDAF, appears to be enriched in the nucleoli. The bodies in which Apoptin and DEDAF are enriched together are most likely different bodies. These bodies may not be present at the same time as nucleoli. Accordingly, some cells in the population will have DEDAF bodies and not nucleoli, whereas other cells in the population will have prominent nucleoli but not DEDAF bodies. This is consistent with the fact that it was difficult to find cells in which
DEDAF bodies and UBF-stained nucleoli were visible simultaneously. Apparently, Apoptin can be enriched in both types of nuclear bodies.

\section{A distinct mutation of Apoptin reduces DEDAF binding and delays apoptosis induction}

Co-localization of Apoptin and DEDAF in tumor cells suggests a biological relevance for their interaction. To investigate this further, we next analyzed by means of co-immunoprecipitations the affinity of a series of Apoptin Alanine replacement mutants for DEDAF. In three independent experiments, the Ala041 mutant containing Alanines at positions 41-45 consistently showed a reduced affinity for DEDAF, whereas other mutants (Ala046, Ala071, Ala076, Ala096, Ala101, and Ala106) showed a lesser affinity in only one or two out of three experiments (Figure 7a and data not shown). Next, we examined the apoptotic activity of the Ala041 mutant in comparison to wild-type Apoptin and the Ala046 mutant, which did not consistently reveal decreased affinity for DEDAF. To that end, Saos-2 cells were transfected with plasmids encoding Ala041, Ala046 or wild-type Apoptin. At 3 or 5 days after transfection, the cells were fixed and examined for induction of apoptosis by means of immunofluorescence. Strikingly, both Ala041 and Ala046 showed a markedly different localization in comparison to wild-type Apoptin. Both proteins revealed a diffuse staining throughout the cell, with Ala046 sometimes present in threadlike structures in the cytoplasm (Figure 7b and c). At 3 days after transfection, the level of apoptosis induced by Ala041 was less than that induced by Ala046 and wild-type Apoptin. However, 5 days after transfection, the level of apoptosis was similar for both Ala-mutants and wild-type Apoptin (Figure 7d). Other Alamutants, which did not consistently show a reduced affinity for DEDAF, hardly had any effect on cell killing as compared to wild-type Apoptin (data not shown). These results suggest that interference with DEDAF-Apoptin interaction delays the induction of apoptosis by Apoptin.

\section{DEDAF induces apoptosis in three human tumor cell lines, but not in mesenchymal stem cells and fibroblasts}

Earlier, DEDAF was found to interact with death effector domains (DED), ${ }^{16}$ such as those found in pro-caspases 8 and 10 , the death receptor adaptor FADD and DEDD (DEDcontaining DNA-binding protein). ${ }^{23}$ DEDAF could be stably expressed in Jurkat and $\mathrm{H} 9$ cells, where it enhanced apoptosis induction by engagement of the death receptor CD95. In HeLa cells, transient expression of DEDAF enhanced apoptosis induced by the overexpressed DEDs of caspase 10 , but had only a minor effect by itself. ${ }^{16}$ These findings suggested that DEDAF could cooperate in general with pro-apoptotic DED-containing proteins, but DEDAF itself was not considered to possess significant pro-apoptotic activity. To further address whether DEDAF contains intrinsic apoptotic activity, we determined the outcome of DEDAF overexpression in normal and tumor cells, and its effect on Apoptin-induced apoptosis. To this end, we expressed 


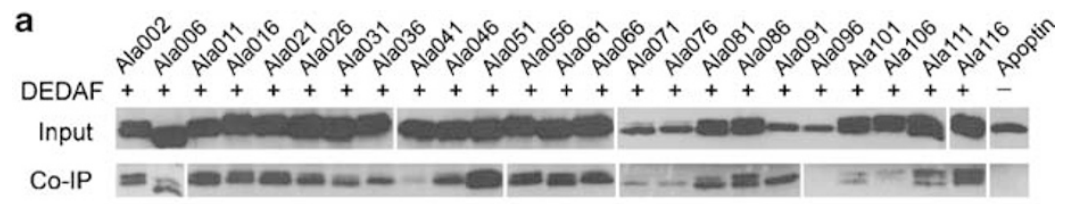

b

Ala041

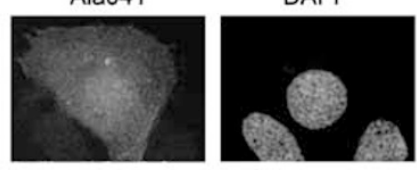

C

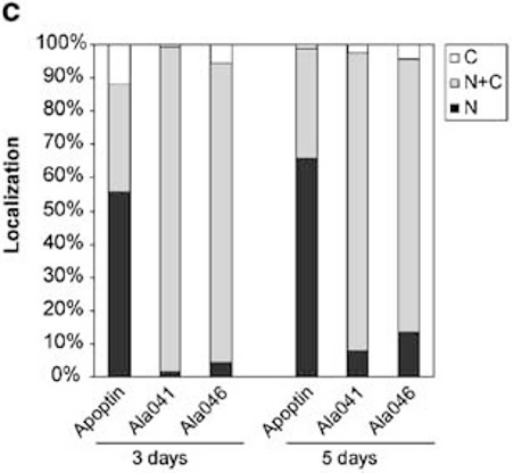

Ala046
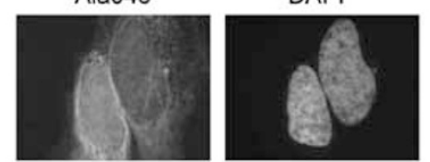

d

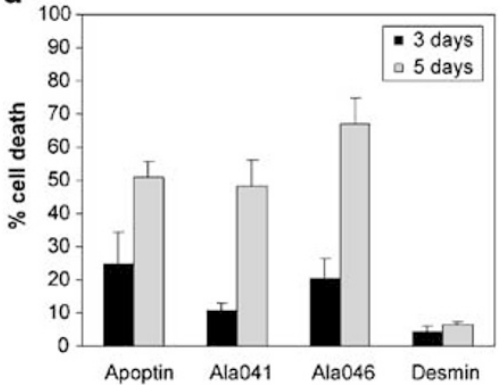

Figure 7 Mutation of a specific part of Apoptin reduces binding to DEDAF and delays apoptosis. (a) Cos cells were transfected with plasmids encoding myc-tagged DEDAF and a series of Alanine replacement mutants of Apoptin, or Apoptin alone as a control. Total lysates (Input) or proteins that co-immunoprecipitated (co-IP) with anti-myc mAb were separated by SDS-PAGE and analyzed by Western blot. Mutant or wild-type Apoptin proteins were detected with R $\alpha$ VP3-C (Ala002-Ala066) or CVICAV-111.3 (Ala071-Ala116 and Apoptin). A representative experiment of three independent experiments is shown. (b) Localization of Ala041 and Ala046 in transiently transfected Saos-2 cells. Proteins were detected by indirect immunofluorescence staining with R $\alpha$ VP3-C. Nuclei were counterstained with DAPI, images were made by fluorescent microscopy. (c, d) Saos-2 cells were transiently transfected with plasmids encoding Apoptin, Ala041, Ala046, or Desmin as a control, and analyzed for protein localization and apoptosis induction by fluorescence microscopy at 3 and 5 days post-transfection. (c) Localization was determined in nonapoptotic cells; $\mathrm{N}$ : nucleus, $\mathrm{N}+\mathrm{C}$ : nucleus + cytoplasm, C: cytoplasm. (d) Apoptosis induction as analyzed by nuclear morphology. Given are the means of three independent experiments; in each experiment, at least 100 positive cells were scored. Error bars represent standard deviation

myc-tagged DEDAF in the tumor cell lines Saos-2, NW18, and SW480, either alone or together with Apoptin, and examined the cells by fluorescence microscopy for expression of the proteins and apoptotic nuclear morphology. Overexpression of DEDAF by itself clearly induced apoptotic morphology in Saos-2, NW18, and SW480 cells (Figure 8a), to similar levels as Apoptin. Co-expression with Apoptin did not significantly increase the percentage of cells with apoptotic nuclear morphology. Similar results were found for DEDAF- $\Delta N 30$ (data not shown).

To determine whether DEDAF-induced cell death is apoptosis, we examined the effect of the caspase inhibitor p35 on DEDAF after co-expression in Saos-2 cells, and analyzed the cells for nuclear apoptotic morphology. p35, which is a broad inhibitor of caspases, ${ }^{24}$ almost completely inhibited the effect caused by overexpression of DEDAF (Figure 8b). Similar results had been found earlier with Apoptin. ${ }^{7}$ Immunofluorescence data provided evidence that p35 has no negative effect on the level of Apoptin or DEDAF in the analyzed cells (data not shown). These results demonstrate the involvement of caspases; therefore, DEDAFinduced cell death can be classified as apoptosis.

To explore further DEDAF's similarities to Apoptin, we determined the effect of DEDAF on normal, untransformed cells. Accordingly, we introduced a DEDAF expression plasmid into human fibroblasts and mesenchymal stem cells via microinjection, and analyzed the cells for nuclear apoptotic morphology 24-48 h later. Desmin was used as a negative control and FADD as a positive control for apoptosis induction. In these normal cell types, DEDAF did not evoke any significant apoptotic response, whereas the cells were sensitive to apoptosis induction by ectopic FADD (Figure 8c). Introducing full-length DEDAF or DEDAF- $\triangle \mathrm{N} 30$ into the normal human fibroblast line $\mathrm{VH} 10$ via transient transfections did not induce apoptosis either (data not shown). Even when we co-expressed DEDAF and Apoptin in human diploid foreskin fibroblasts, no induction of cell death could be observed. In the tumor cell line Saos-2, microinjection of DEDAF resulted in significant apoptosis $48 \mathrm{~h}$ later (data not shown). Taken together, DEDAF has similar properties as Apoptin, in that, upon overexpression, DEDAF induced cell death in tumor cells via a mechanism that is dependent on caspases, but overexpression did not appear to harm normal, untransformed cells.

\section{Discussion}

In this study, we show that human DEDAF specifically interacts with viral Apoptin. Mutation of a specific part of Apoptin resulted in a strongly reduced binding and delayed apoptosis. Recently, we have shown that Apoptin contains 


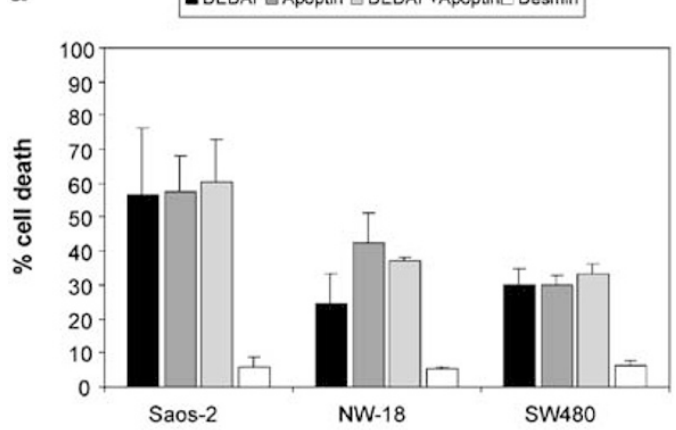

b

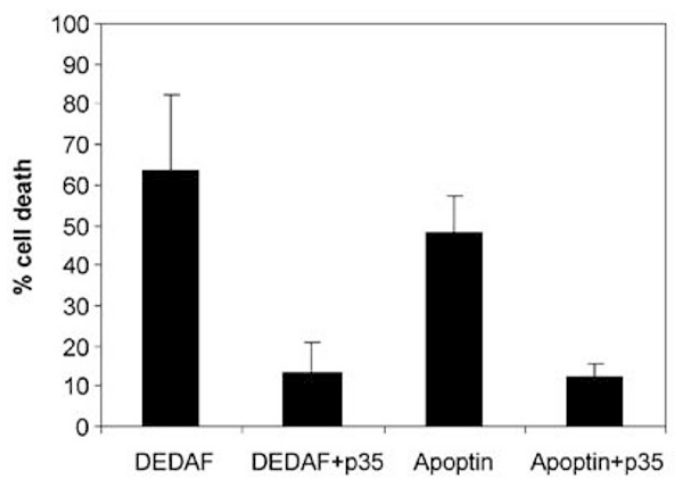

C

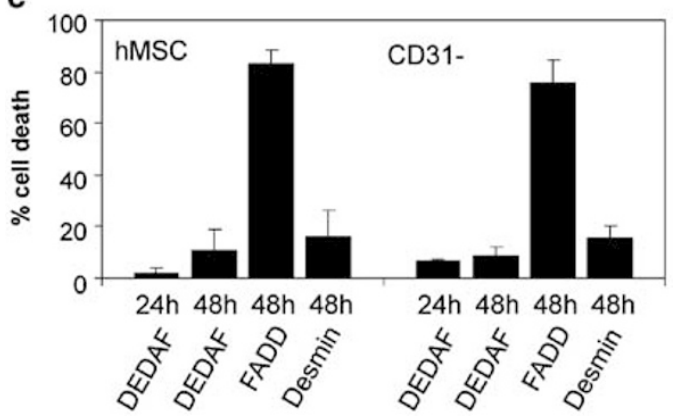

Figure 8 Apoptosis induction by DEDAF. (a) Saos-2, NW-18, and SW480 cells were transfected with plasmids encoding DEDAF and Apoptin alone or in combination, or with Desmin as a control. At day 5 post-transfection, cells were fixed, stained with specific antibodies and analyzed by fluorescence microscopy for protein expression and nuclear morphology, as detected by DAPI staining. The percentage of cells displaying apoptotic nuclear morphology among those expressing the proteins of interest was scored. Data shown are the means of multiple independent experiments (Saos-2: $n=10, N W-18: n=3$, SW480: $n=2$ ); error bars represent standard deviations. (b) Saos-2 cells were transfected with plasmids encoding DEDAF or Apoptin, combined with an equal amount of plasmid encoding p35, or empty vector as a control. Analysis was done as indicated for panel (a). Data given are the means of five independent experiments; error bars represent standard deviations. (c) Diploid foreskin fibroblasts and mesenchymal stem cells were microinjected with plasmids encoding DEDAF, FADD as a positive control or Desmin as a negative control. Dextran-rhodamine was co-injected as a marker. Cells were fixed 24 or $48 \mathrm{~h}$ posttransfection and analyzed by indirect immunofluorescence. The percentage of apoptotic cells among those containing dextran-rhodamine was determined by evaluating the nuclear morphology. Values given are the means of multiple independent experiments (FADD: $n=2$, DEDAF: $n=3$, Desmin: $n=3$ ), error bars represent the standard deviations

two different domains that induce apoptosis independently, ${ }^{13}$ which might explain why we only observed a delayed apoptosis induction when the DEDAF/Apoptin interaction was prevented. DEDAF was isolated earlier in a yeast twohybrid screen, using the DED of pro-caspases 8 and $10,{ }^{16}$ and was also found by others as a binding partner for the transcription factor E4TF1/hGABP and named YEAF1. ${ }^{17}$ RYBP, its murine homologue, was isolated in a yeast twohybrid screen with Ring1A, a member of the Polycomb group of transcription regulators. ${ }^{18}$ DEDAF and RYBP have a close structural and functional homologue, termed YAF-2. ${ }^{19} \mathrm{DE}$ DAF has also been found to interact with members of the E2F transcription factor family. ${ }^{25}$

The open reading frame of the full-length DEDAF cDNA encodes a protein of 226 amino acids with an estimated molecular mass of $25 \mathrm{kDa}$. Amino acids $25-42$ contain a $\mathrm{C}_{2} \mathrm{C}_{2}$-type zinc-finger motif, while two putative NLSs are located at amino acids $76-79$ and $102-108$. The NLS at amino acids $76-79$ is fully conserved among all the three proteins, while the second NLS is the same in DEDAF and RYBP and differs by two amino acids in YAF2. The zinc-finger motif is fully conserved among all the three proteins, with the exception of two residues C-terminal of the last cysteine in the motif of YAF2. The N-terminal part including the zincfinger is not required for binding to $\mathrm{YY} 1$ or Polycomb group proteins ${ }^{18,19}$ The proteins further share a high content of basic residues ( 41 out of 226 for both DEDAF and RYBP) and an abundance of serine and threonine residues in the $\mathrm{C}$-terminus (31 out of 84 in both proteins). Although there is no apparent sequence homology between these proteins and Apoptin, they do share the presence of two NLS sequences, a high content of basic residues, and an abundance of serines and threonines.

In agreement with others, we found that DEDAF is broadly expressed at the mRNA level, but we could not confirm reports finding high expression in human hematopoietic tissues. One possible reason for this discrepancy may be that, unlike Zheng et al., ${ }^{16}$ we analyzed transcripts from thymus and spleen on the same blot as those from other tissues. There is consensus about high expression in placenta, which contains a mixture of adult and fetal cells. In tissues from 22-37-weekold embryos, however, we did not find enhanced levels of the transcript, suggesting that the high expression in placenta is tissue specific, and not due to the fetal component. For RYBP, in situ hybridization of mouse embryos revealed selective expression in cells of the developing nervous system at day E9.0, which points to a role during embryonal development. However, RYBP was expressed ubiquitously from day E9.5 onwards. ${ }^{18}$ The broad expression of DEDAF/RYBP is in line with a functional interaction with Apoptin, which has the capacity to induce apoptosis in a great variety of tumor cell lines, irrespective of their tissue of origin.

There is agreement in the literature that overexpressed DEDAF/RYBP is predominantly present in the nucleus, but this has thus far only been tested in transfected cells. We observed a similar staining, but analysis of endogenous protein will be required to determine whether this localization is physiological. Unfortunately, our antibody was unable to detect endogenous protein in either Western blot or immunocytochemical analysis, although the antibody was very powerful in detecting exogenously expressed DEDAF in both techniques. This implies that endogenous DEDAF may be present in very low amounts. 
We find here that DEDAF can act as a transcriptional repressor when tethered to a promoter. This result is consistent with the finding that its murine homologue RYBP can act as a repressor in a comparable system. ${ }^{18} \mathrm{DEDAF} /$ RYBP may therefore be responsible for or contribute to the repressor activity of $Y Y 1$, to which it can bind. As suggested for DEDD, the role of DEDAF in apoptosis may be to inhibit the transcription of certain genes. Interestingly, Apoptin has been found to bind to DNA and colocalize with heterochromatin, which is predominantly transcriptionally inactive. ${ }^{26}$ Similar to DEDD, Apoptin appears to be regulated by translocation to the nucleus, although nuclear localization of Apoptin is not sufficient to induce apoptosis and its phosphorylation appears to be required as well. ${ }^{14}$ The finding that Apoptin binds to DEDAF is interesting, because it hints at a possible role of transcription repression in Apoptininduced apoptosis. Recently, we have shown that inhibitors of transcription or translation do not affect Apoptin's activity; however, this does not exclude a function in transcriptional repression. ${ }^{13}$

Overexpression of DEDAF induced apoptosis in three different tumor cell lines, but not in normal fibroblasts or mesenchymal stem cells. In contrast, FADD overexpression did induce apoptosis in these normal cells, indicating that the cells were competent to execute apoptosis. DEDAF- $\Delta N 30$ behaved the same as full-length DEDAF, showing that the $\mathrm{N}$ terminal amino acids are not required for apoptosis induction. It is intriguing that DEDAF appears to have a similar preference for tumor cells as Apoptin. These data are consistent with a model whereby DEDAF and Apoptin induce cell death by the same mechanism.

Although we were unable to detect endogenous DEDAF using a specific antibody, Northern blotting suggested a broad expression pattern, including in tumor cell lines. Thus, endogenous DEDAF does not seem to harm tumor cells, which implies that there may be a threshold level below which DEDAF does not induce apoptosis. Alternatively, tumor cells may have accumulated genetic lesions that inhibit DEDAF activity, such as upregulation of an apoptosis inhibitor. In our hands, titrating the amount of DEDAF DNA down six-fold, until reaching the detection limit, did not result in diminished cell death (data not shown). Stable transfectants of RYBP in human U2OS tumor cells ${ }^{18}$ and of DEDAF in Jurkat and $\mathrm{H} 9$ cells ${ }^{16}$ have been obtained, but may have been selected for low expression levels, as suggested by Garcia et al. ${ }^{18}$ Furthermore, Zheng et al. ${ }^{16}$ have observed some cell death when DEDAF was transiently expressed in HeLa cells, which supports our finding that overexpression of DEDAF results in apoptosis of tumor cells.

In addition, we have shown here that DEDAF binds to the viral apoptosis agonist Apoptin. Furthermore, DEDAF not only partially co-localized with Apoptin, but also displayed similar cell-killing activity when overexpressed in tumor cells. Moreover, DEDAF appeared not to induce cell death in normal, untransformed cells. It will be interesting to determine whether Apoptin acts through DEDAF in mammalian cells, and to further examine DEDAF's tumor-preferential cell-killing activity. In the future, DEDAF may prove to be an appropriate human, nonimmunogenic apoptosis agonist for exploitation in cancer therapy.

\section{Materials and Methods}

\section{Yeast two-hybrid screen}

For the yeast two-hybrid screen, we used a cDNA library derived from Epstein-Barr virus-transformed human lymphocytes, which was cloned into the leucine-selectable PACT yeast expression vector, as described by Durfee et al. ${ }^{15}$ The cDNAs were inserted into the Xhol site downstream of the gene encoding the GAL4 activation domain (AD) in pACT. Full-length Apoptin was cloned downstream of the gene encoding the GAL4 DNAbinding domain (BD) in the tryptophan-selectable plasmid pGBT9 (Clontech) and confirmed by sequencing. This plasmid, pGBT9-Apoptin, which expresses the fusion protein GAL4-BD-Apoptin, was used as bait in the yeast two-hybrid screen. The yeast strain Y190, which is $\mathrm{LeU}^{-}$and $T_{r p}{ }^{-}$, and contains LacZ and HIS3 reporter genes for GAL4 transcriptional activity, was transformed with pGBT9-Apoptin according to the Klebe protocol. ${ }^{27}$ In a $\beta$ Gal colony filter assay, ${ }^{28}$ the bait plasmid was shown to have no transactivation activity on its own. Next, the cDNA library was transformed into Y190 containing pGBT9-Apoptin, with an efficiency of $1 \times 10^{6}$, and grown on plates lacking Leu, Trp and His. His ${ }^{+}$colonies were tested for $\beta$ Gal activity by colony filter assay, and double-positive colonies $\left(\mathrm{His}^{+} / \mathrm{LacZ}^{+}\right.$) were selected for rescue of library plasmids on $E$. coli strain DH10B. Library plasmids were retransformed into $Y 190$ either alone, or together with pGBT9-Apoptin or pGBT9. A $\beta$ Gal colony filter assay was performed to discern bona fide Apoptin-binders from GAL4-BD binders or GAL4 site binders. The resulting clones were sequenced by Eurogentec.

\section{Northern blot hybridization}

The human 12-lane multiple tissue Northern (MTN) blot, which contains mRNA derived from 12 different normal tissues, and the human cancer cell line MTN blot, with mRNA from eight different tumor cell lines, were both acquired from Clontech. The human fetal mRNA Northern blot contains purified mRNA from four different fetal tissues (age 22-37 weeks) and from adult lung (Invitrogen). All blots contain approximately $2 \mu \mathrm{g}$ of polyA ${ }^{+}$RNA per lane. The blots were pre-hybridized for $4 \mathrm{~h}$ at $42^{\circ} \mathrm{C}$ in $10 \mathrm{ml}$ of pre-hybridization mix $(5 \times$ SSC, $5 \times$ Denhardts, $1 \%$ SDS, and $50 \%$ formamide). For the probe, the library plasmid containing clone 40 was digested with $\mathrm{Xhol}$, which generates a fragment encoding DEDAF with a 30 aa $\mathrm{N}$-terminal deletion (DEDAF $\triangle N 30$ ). This fragment was purified by agarose gel isolation with the Jetsorb kit (Genomed). A DNA fragment encoding $\beta$-actin was used as a control for equal loading. Subsequently, the probes were labeled with $\alpha^{32} \mathrm{P}$-dATP according to the mixed primer-labeling protocol. ${ }^{29}$ Free label was removed by G50 Sepharose gel filtration spin columns. For the hybridization, $100 \mathrm{ng}$ of labeled probe was boiled together with $100 \mu \mathrm{g}$ of salmon sperm DNA and added to the blots. Hybridization was carried out overnight at $42^{\circ} \mathrm{C}$. Finally, the blots were washed with $2 \times \mathrm{SSC} / 0.05 \%$ SDS (three times $10 \mathrm{~min}$ at room temperature) and $0.1 \times \mathrm{SSC} / 0.1 \% \mathrm{SDS}$ (two times $15 \mathrm{~min}$ at $50^{\circ} \mathrm{C}$ ) and exposed to an X-ray film.

\section{Plasmids}

Positive clones from the yeast two-hybrid screen were digested with Xhol to generate cDNA fragments, which were subcloned into pMT2SM-myc, ${ }^{30}$ providing the fragments with an in-frame $\mathrm{N}$-terminal myc-tag (9E10) and conferring mammalian expression under the control of the MLP promoter. pCMV-VP3, encoding Apoptin, pCMV-Neo-Bam, the empty control plasmid, pCMV-Desmin, encoding Desmin, pCMV-p35, encoding p35, 
and pCMV-CrmA, encoding CrmA, have all been described before. ${ }^{5,31}$ pcDNA3.1/mycHis/LacZ (Invitrogen) encodes LacZ with a myc and His tag attached to the $\mathrm{C}$-terminus, regulated by a CMV promoter. The plasmid pcDNA3-HA-FADD was kindly provided by $V$ Dixit and the University of Michigan, and has been described previously by Chinnaiyan et al. ${ }^{32}$ pCMV-HA/YY1 expressing HA-tagged $Y Y 1$ and pGal4-YY1 encoding $Y Y 1$ fused to the Gal4 DNA-BD were kind gifts from $Y$ Shi and $R$ Johnstone. ${ }^{33}$ pFA-CMV (Stratagene) contains the Gal4 BD upstream of a multiple cloning site, under the regulation of a CMV promoter. DNA fragments encoding DEDAF or Apoptin were cloned into the multiple cloning site of pFA-CMV, and confirmed by sequencing (Baseclear). The resulting plasmids pFA-AAP1 and pFA-VP3 encode Gal4 BD fusions to DEDAF and Apoptin, respectively. The reporter plasmid $5 \times$ Gal4-TK-Luc was a kind gift from $E$ Kalkhoven, and contains the luciferase gene under the regulation of the herpes simplex virus TK promoter joined to $5 \times \mathrm{Gal} 4$ binding elements. ${ }^{34}$ All plasmids were purified with Jetstar (Genomed) or Qiagen (Qiagen) maxiprep columns.

A panel of Apoptin Ala-mutants was kindly provided by D Mumberg. These mutants were constructed by serial replacement of five amino-acid residues of Apoptin with Alanine residues. To this end, first, an Apoptin DNA containing additional unique restriction enzyme sites that allow for ease of cloning was constructed (SAPO). Then Apoptin amino-acid residues were exchanged by five Alanine residues using a linker substitution strategy. The Ala-mutants and SAPO were sequenced and cloned in a modified expression plasmid vector pIRESneo (Clontech) under the control of the CMV promoter.

\section{Cell culture}

The human osteosarcoma cell lines Saos-2 and U2OS, the SV40transformed monkey kidney cell line Cos-1, and the human normal diploid skin fibroblast strain VH10 (up to passage 14) were all grown in DMEM, supplemented with $10 \%$ fetal bovine serum (FBS). NW-18, human SV40transformed tumorigenic fibroblasts, were grown in MEM supplemented with $8 \%$ FBS. SW480, human colorectal adenocarcinoma cells (ATCC), were grown in Leibovitz's L-15 medium supplemented with $2 \mathrm{mM}$ Lglutamine and $10 \%$ FBS. Low-passage normal diploid foreskin fibroblasts, kindly provided by Schering AG (Berlin), were grown in MEM-Earle (Biochrom KG) supplemented with 10\% FBS. Human mesenchymal stem cells (hMSC) were purchased from BioWhittaker and cultured according to their protocols for no more than four passages. All culture media were obtained from Life Technologies Inc. unless mentioned otherwise, and contained penicillin and streptomycin.

\section{Transfection and microinjection methods}

Saos-2, U2OS, and Cos-1 cells were transfected by the $\mathrm{CaPO}_{4} \mathrm{co}-$ precipitation method with $3 \mu \mathrm{g}$ DNA per well of a six-well dish, or $20 \mu \mathrm{g}$ DNA per $9 \mathrm{~cm}$ dish. NW-18, SW480, and VH10 cells were transfected with FUGENE 6 (Roche Molecular Biochemicals) according to the manufacturer's protocol; $2 \mu \mathrm{g}$ DNA was transfected per chamber or 3-6 $\mu \mathrm{g}$ DNA per well of a six-well dish. For microinjections of diploid foreskin fibroblasts or hMSC, cells were cultured on glass-bottomed microinjection dishes (MatTek Corporation) or in $35 \mathrm{~mm}$ dishes containing glass Cellocate coverslips (Eppendorf). The cells were microinjected in the nucleus with DNA (100 ng/ $\mu$ l in PBS) using an Eppendorf microinjector with the injection-pressure condition of $0.5 \mathrm{psi}$. The cells were co-injected with dextran-rhodamine (MW 70 kDa; Molecular Probes) to be able to later identify the injected cells. At least 100 cells were microinjected per sample, of which 20-80 were found to express protein when analyzed.

\section{Immunoprecipitation (IP) assay and Western blot analysis}

For co-immunoprecipitation of myc-tagged DEDAF with Apoptin, or with YY1, Cos-1 cells were lysed 40-44 h after transfection. Cells were washed with cold PBS, scraped in $400 \mu$ lysis buffer ( $50 \mathrm{mM}$ Tris pH 7.5, $250 \mathrm{mM}$ $\mathrm{NaCl}, 5 \mathrm{mM}$ EDTA, $0.1 \%$ Triton X-100, $20 \mathrm{mM} \beta$-glycerophosphate, $5 \mathrm{mM}$ $\mathrm{NaF}, 1 \mathrm{mM}$ PMSF, $1 \mu \mathrm{g} / \mathrm{ml}$ leupeptin, $200 \mu \mathrm{g} / \mathrm{ml}$ trypsin inhibitor, $1 \mu \mathrm{g} / \mathrm{ml}$ pepstatin), transferred to a microfuge tube and kept on ice for $30 \mathrm{~min}$. Cell debris was removed by centrifugation at $13000 \times g, 4^{\circ} \mathrm{C}$, for $10 \mathrm{~min}$. For each sample, $40 \mu \mathrm{l}$ of $10 \%$ Protein A-sepharose beads (Sigma-Aldrich) were coupled to mouse monoclonal anti-myc antibody (9E10; Pharmingen), rabbit polyclonal anti-Apoptin antibody $\mathrm{R} \alpha \mathrm{VP} 3-\mathrm{C}^{7}$, or rabbit polyclonal antibody against YY1 (sc 1703; Santa Cruz Biotechnology) and incubated with the lysate for 2-3 h. Next, the beads were washed three times with lysis buffer and once with PBS and boiled in Laemmli sample buffer. Samples were loaded along with the total lysates onto $12.5 \%$ SDS polyacrylamide gels, separated by electrophoresis, and electroblotted onto Immobilon-P membranes (Millipore). Blots were incubated with anti-myc antibody 9E10 to detect DEDAF and LacZ, with CVI-CAV- $111.3^{8}$ to detect Apoptin, or with mouse mAb anti-HA (HA.11; Covance) to detect YY1. Signals were visualized by enhanced chemiluminescence, according to the manufacturer's protocol (Amersham).

\section{Immunofluorescence microscopy}

At different time points post-transfection, cells grown on glass cover slips were fixed with $50 \%$ acetone $/ 50 \%$ methanol for $5 \mathrm{~min}$, air-dried, and stored at $-20^{\circ} \mathrm{C}$ until further use. VH10 cells, which were grown on Permanox chamber slides (Life Technologies), were fixed by the same method. Cells that were microinjected with dextran-rhodamine were fixed with $1 \%$ formaldehyde for $10 \mathrm{~min}$, followed by $100 \%$ methanol for $5 \mathrm{~min}$, and $80 \%$ acetone for $3 \mathrm{~min}$. Indirect immunofluorescence assays were performed as described. ${ }^{7}$ Apoptin was detected with the rabbit polyclonal antibody $\mathrm{R} \alpha \mathrm{VP3}-\mathrm{C}^{7}$ and DEDAF or LacZ were detected with anti-myc antibody (9E10). FADD was detected with mouse mAb anti-FADD (BD Transduction Laboratories), and Desmin was detected with mouse $\mathrm{mAb}$ 33 (Monosan). UBF was stained with rabbit polyclonal anti-UBF (H300; sc9131, Santa Cruz Biotechnology). Secondary antibodies were either conjugated to fluorescein isothiocyanate (FITC) or Rhodamine (Jackson ImmunoResearch Laboratories). DNA was stained with $1 \mu \mathrm{g} \mathrm{2,4-}$ diamidino-2-phenylindole (DAPI) per ml. The cells were analyzed by fluorescence microscopy (AX70 Provis; Olympus) for expression of the transfected protein. Nuclear morphology indicating the apoptotic state of the cell was determined by examining the DAPI staining. In apoptotic cells, the chromatin is condensed, the nucleus is often fragmented, and finally DAPI staining is lost due to fragmentation of the DNA. At least 100 cells were scored per sample in each experiment. Images of representative cells were made by confocal laser-scanning microscopy (TCS; Leica Microsystems).

\section{Luciferase reporter assay}

U2OS cells were plated in six-well plates and transfected with FUGENE 6 (Roche Molecular Biochemicals) and with $1 \mu \mathrm{g} /$ well $5 \times$ Gal4-TK-Luc as the reporter plasmid, pcDNA3.1/mycHis/LacZ at $100 \mathrm{ng} /$ well as an internal control, and $\mathrm{Gal} 4 \mathrm{BD}$ plasmids at $200 \mathrm{ng} /$ well or at varying amounts. After 2 days, cells were lysed in Reporter Lysis Buffer (Promega) and lysates 
were assayed for luciferase activity and for $\beta$-galactosidase activity, according to the manufacturer's protocol (Promega).

\section{Acknowledgements}

We thank Drs. Yang Shi and Ricky Johnstone for donation of pCMV-HA/ YY1 and pGal4-YY1, Dr. Eric Kalkhoven for the plasmid $5 \times$ Gal4-TK-Luc and advice on the reporter assay, Dr. Dominik Mumberg for providing the Apoptin Ala-mutants, Drs. Hans van Ormondt and Bertram Weiss for their help with sequence analysis, and Stefan Erkeland for technical assistance. We further thank Schering AG for providing diploid foreskin fibroblasts, and Dr. Vishva Dixit and the University of Michigan for the plasmid pcDNA3-HA-FADD. This work was supported by grants from the Dutch Cancer Society, the Netherlands Ministry of Economic Affairs and Schering AG.

\section{References}

1. Hanahan D and Weinberg RA (2000) The hallmarks of cancer. Cell 100:57-70

2. Lowe SW, Bodis S, McClatchey A, Remington L, Ruley HE, Fisher DE, Housman DE and Jacks T (1994) p53 status and the efficacy of cancer therapy in vivo. Science 266: 807-810

3. Reed JC (1995) Regulation of apoptosis by bcl-2 family proteins and its role in cancer and chemoresistance. Curr. Opin. Oncol. 7: 541-546

4. Danen-Van Oorschot AAAM, Zhang Y, Erkeland SJ, Fischer DF, van der Eb AJ and Noteborn MHM (1999) The effect of Bcl-2 on Apoptin in 'normal' vs transformed human cells. Leukemia 13 (Suppl 1): S75-S77

5. Noteborn MHM, Todd D, Verschueren CA, de Gauw HW, Curran WL, Veldkamp S, Douglas AJ, McNulty MS, Van der Eb AJ and Koch G (1994) A single chicken anemia virus protein induces apoptosis. J. Virol. 68: 346-351

6. Zhuang SM, Landegent JE, Verschueren CA, Falkenburg JH, van Ormondt $\mathrm{H}$, van der Eb AJ and Noteborn MHM (1995) Apoptin, a protein encoded by chicken anemia virus, induces cell death in various human hematologic malignant cells in vitro. Leukemia 9 (Suppl 1): S118-S120

7. Danen-van Oorschot AAAM, van der Eb AJ and Noteborn MHM (2000) The chicken anemia virus-derived protein apoptin requires activation of caspases for induction of apoptosis in human tumor cells. J. Virol. 74: 7072-7078

8. Danen-Van Oorschot AAAM, Fischer DF, Grimbergen JM, Klein B, Zhuang S, Falkenburg JH, Backendorf C, Quax PH, van der Eb AJ and Noteborn MHM (1997) Apoptin induces apoptosis in human transformed and malignant cells but not in normal cells. Proc. Natl. Acad. Sci. USA 94: 5843-5847

9. Noteborn MHM (2002) Apoptin as an anticancer therapy. In Targets for Cancer Chemotherapy La Thangue NB and Bandara LR (eds) (Totowa, NJ: Humana Press) pp. 275-287

10. Zhang YH, Abrahams PJ, van der Eb AJ and Noteborn MHM (1999) The viral protein Apoptin induces apoptosis in UV-C-irradiated cells from individuals with various hereditary cancer-prone syndromes. Cancer Res. 59: 3010-3015

11. Noteborn MHM, Zhang YH and van der Eb AJ (1998) Apoptin specifically causes apoptosis in tumor cells and after UV-treatment in untransformed cells from cancer-prone individuals: a review. Mutat. Res. 400: 447-455

12. Zhuang SM, Shvarts $A$, van Ormondt $H$, Jochemsen AG, van der Eb AJ and Noteborn MHM (1995) Apoptin, a protein derived from chicken anemia virus, induces p53-independent apoptosis in human osteosarcoma cells. Cancer Res. 55: 486-489

13. Danen-van Oorschot AAAM, Zhang Y-H, Leliveld SR, Rohn JL, Seelen MCMJ, Bolk MW, van Zon A, Erkeland SJ, Abrahams J-P, Mumberg D and Noteborn MHM (2003) Importance of nuclear localization of Apoptin for tumor-specific induction of apoptosis. J. Biol. Chem. 278: 27729-27736

14. Rohn JL, Zhang YH, Aalbers RI, Otto N, Den Hertog J, Henriquez NV, Van De Velde CJ, Kuppen PJ, Mumberg D, Donner P and Noteborn MHM (2002) A tumor-specific kinase activity regulates the viral death protein apoptin. J. Biol. Chem. 277: 50820-50827
15. Durfee T, Becherer K, Chen PL, Yeh SH, Yang Y, Kilburn AE, Lee WH and Elledge SJ (1993) The retinoblastoma protein associates with the protein phosphatase type 1 catalytic subunit. Genes Dev. 7: 555-569

16. Zheng L, Schickling O, Peter ME and Lenardo MJ (2001) The death effector domain-associated factor plays distinct regulatory roles in the nucleus and cytoplasm. J. Biol. Chem. 276: 31945-31952

17. Sawa C, Yoshikawa T, Matsuda-Suzuki F, Delehouzee S, Goto M, Watanabe $\mathrm{H}$, Sawada J, Kataoka K and Handa H (2002) YEAF1/RYBP and YAF-2 are functionally distinct members of a cofactor family for the YY1 and E4TF1/ hGABP transcription factors. J. Biol. Chem. 277: 22484-22490

18. Garcia E, Marcos-Gutierrez C, del Mar L, Moreno JC and Vidal M (1999) RYBP, a new repressor protein that interacts with components of the mammalian Polycomb complex, and with the transcription factor YY1. EMBO J. 18: 3404-3418

19. Kalenik JL, Chen D, Bradley ME, Chen SJ and Lee TC (1997) Yeast two-hybrid cloning of a novel zinc finger protein that interacts with the multifunctional transcription factor YY1. Nucleic Acids Res. 25: 843-849

20. Kozak M (1984) Compilation and analysis of sequences upstream from the translational start site in eukaryotic mRNAs. Nucleic Acids Res. 12: 857-872

21. Shrivastava A, Saleque S, Kalpana GV, Artandi S, Goff SP and Calame K (1993) Inhibition of transcriptional regulator Yin-Yang-1 by association with c-Myc. Science 262: 1889-1892

22. Chan EK, Imai H, Hamel JC and Tan EM (1991) Human autoantibody to RNA polymerase I transcription factor hUBF. Molecular identity of nucleolus organizer region autoantigen NOR-90 and ribosomal RNA transcription upstream binding factor. J. Exp. Med. 174: 1239-1244

23. Stegh AH, Schickling O, Ehret A, Scaffidi C, Peterhansel C, Hofmann TG, Grummt I, Krammer PH and Peter ME (1998) DEDD, a novel death effector domain-containing protein, targeted to the nucleolus. EMBO J. 17: 5974-5986

24. Zhou Q, Krebs JF, Snipas SJ, Price A, Alnemri ES, Tomaselli KJ and Salvesen GS (1998) Interaction of the baculovirus anti-apoptotic protein p35 with caspases. Specificity, kinetics, and characterization of the caspase/p35 complex. Biochemistry 37: 10757-10765

25. Schlisio S, Halperin T, Vidal M and Nevins JR (2002) Interaction of YY1 with E2Fs, mediated by RYBP, provides a mechanism for specificity of E2F function. EMBO J. 21: $5775-5786$

26. Leliveld SR, Dame RT, Mommaas MA, Koerten HK, Wyman C, Danen-van Oorschot AAAM, Rohn JL, Noteborn MHM and Abrahams JP (2003) Apoptin protein multimers form distinct higher-order nucleoprotein complexes with DNA. Nucleic Acids Res. 31: 4805-4813

27. Klebe RJ, Harriss JV, Sharp ZD and Douglas MG (1983) A general method for polyethylene-glycol-induced genetic transformation of bacteria and yeast. Gene 25: 333-341

28. Chevray PM and Nathans D (1992) Protein interaction cloning in yeast: identification of mammalian proteins that react with the leucine zipper of Jun. Proc. Natl. Acad. Sci. USA 89: 5789-5793

29. Feinberg AP and Vogelstein B (1984) A technique for radiolabeling DNA restriction endonuclease fragments to high specific activity. Addendum Anal. Biochem. 137: 266-267

30. Gebbink MF, Kranenburg O, Poland M, van Horck FP, Houssa $B$ and Moolenaar WH (1997) Identification of a novel, putative Rho-specific GDP/GTP exchange factor and a RhoA-binding protein: control of neuronal morphology. J. Cell. Biol. 137: 1603-1613

31. Zhuang SM, Shvarts A, Jochemsen AG, van Oorschot AAAM, van der Eb AJ and Noteborn MHM (1995) Differential sensitivity to Ad5 E1B-21kD and Bcl-2 proteins of apoptin-induced versus p53-induced apoptosis. Carcinogenesis 16 : 2939-2944

32. Chinnaiyan AM, O'Rourke K, Tewari M and Dixit VM (1995) FADD, a novel death domain-containing protein, interacts with the death domain of Fas and initiates apoptosis. Cell 81: 505-512

33. Shi Y, Seto E, Chang LS and Shenk T (1991) Transcriptional repression by YY1, a human GLI-Kruppel-related protein, and relief of repression by adenovirus E1A protein. Cell 67: 377-388

34. Kalkhoven E, Teunissen H, Houweling A, Verrijzer CP and Zantema A (2002) The PHD type zinc finger is an integral part of the CBP acetyltransferase domain. Mol. Cell. Biol. 22: 1961-1970 